\title{
Effect of Cisplatin on In Vitro Production of Lipid Peroxides in Rat Kidney Cortex
}

\author{
Kumi SUGIHARA, Sachiko NAKANO and Munekazu GEMBA* \\ Division of Pharmacology. Osaka University of Pharmaceutical Sciences, \\ Kawai, Matsubara, Osaka 580, Japan \\ Accepted February 2, 1987
}

\begin{abstract}
Cisplatin (cis-diamminedichloroplacinum II), an antitumor agent with a dose-limiting adverse effect of nephrotoxicity, increased lipid peroxidation in a time- and concentration-dependent manner in rat renal slices incubated in vitro. The addition of an antioxidant, $N$ - $N^{\prime}$-diphenyl-p-phenylenediamine (DPPD), to the incubation medium completely inhibited this increase. We also studied the in vitro effects of agents that modify cisplatin nephrotoxicity on lipid peroxidation in the slices caused by cisplatin. Mannitol, which protects against cisplatin nephrotoxicity, almost completely inhibited the increase in lipid peroxidation caused by cisplatin. Methionine, which potentiates cisplatin nephrotoxicity, made the slices more susceptible to peroxidation. The decrease with cisplatin in $p$-aminohippurate (PAH) accumulation in incubated kidney cortical slices, the accumulation being a representative biochemical process in the transport ability of renal cells, was partially inhibited when DPPD was in the medium. The results suggested that cisplatin directly affected renal tissues in which free radicals generated by cisplatin may interact with membrane lipids to cause the production of lipid peroxides that damage membrane function. Compounds that modify cisplatin nephrotoxity such as mannitol and methionine may act by affecting the production of renal lipid peroxides by cisplatin.
\end{abstract}

Cisplatin (cis-diamminedichloroplatinum II) is an important chemotherapeutic agent. It is highly effective in the treatment of ovarian. testicular, and bladder carcinomas, cancers of the head and neck, and elsewhere (1). The most important adverse effect of cisplatin. which limits doses that can be used, is nephrotoxicity (2-5). The mechanism responsible for ihis nephrotoxicity is not known. Orgotein which has superoxide dismutase activity ameliorates the nephrotoxicity of cisplatin (6). Pretreatment of rats with an antioxidant, $\alpha$-tocopherol or $N-N^{\prime}$ diphenyl-p-phenylenediamine (DPPD), decreases the cisplatin-induced nephrotoxicity (7). These results suggest that the production of free radicals may be responsible for the nephrotoxicity. The injection of cisplatin to rats increases lipid peroxides in the kidney

\footnotetext{
* To whom all correspondence should be addressed.
}

(8). Abnormal levels of free radicals react with polyunsaturated fatty acids and cause an increase in lipid peroxides $(9,10)$. An increase in the lipid peroxidation of polyunsaturated fatty acids in plasma and subcellular membranes may cause cellular disturbances. Lipid peroxidation has been implicated in the development of renal injury caused by cephaloridine, mercuric chloride. gentamicin, ischemia and reflow (11-14).

In this study, we examined the effects of cisplatin on lipid peroxidation in renal cortical slices and measured $p$-aminohippurate (PAH) accumulation in the slices as a biochemical index for an in vitro evaluation of cellular damage. We investigated whether DPPD, a powerful antioxidant $(15,16)$, had an effect on changes in lipid peroxidation and PAH accumulation caused by cisplatin. We reported that cisplatin caused a significant increase in lipid peroxidation and a decrease 
in PAH accumulation in the renal cortical slices in vitro and that the antioxidant inhibited these changes.

\section{Materials and Methods}

Preparation of renal slices: Male SpragueDawley rats weighing about $200 \mathrm{~g}$ were used. They were fed standard chow and given free access to water. The rats were decapitated, and the kidneys were rapidly removed, decapsulated and placed in icecold isotonic saline medium $(0.9 \% \mathrm{NaCl})$. Thin renal slices $(0.3-0.4 \mathrm{~mm}$ in thickness) were prepared free-hand with a razor blade on an ice-cold Petri dish. The slices were immersed in the chilled saline medium until use.

Measurement of lipid peroxidation in the slices: Renal slices weighing about $200 \mathrm{mg}$ were incubated at $37^{\circ} \mathrm{C}$ in $5 \mathrm{ml}$ of incubation medium containing $150 \mathrm{mM} \mathrm{KCl}$ and $20 \mathrm{mM}$ Tris- $\mathrm{HCl}$ buffer, $\mathrm{pH}$ 7.4, with or without cisplatin and another agent such as DPPD. The measurement of lipid peroxidation using renal slices was conducted in Tris- $\mathrm{HCl}$ buffer rather than Hepes buffer. The reason for this is that the slices incubated in the former had a lower base-line value for lipid peroxides than the slices incubated in the latter, although the production of lipid peroxides in the incubated slices were affected by cisplatin to the same extent in both Tris- $\mathrm{HCl}$ buffer and Hepes buffer. After incubation, the slices were removed, weighed, and homogenized in the same medium. Lipid peroxidation in homogenates prepared from renal tissues was monitored by the measurement of production of malondialdehyde with the thiobarbituric acid assay described by Buege and Aust (17).

PAH accumulation during the incubation of the slices: The experimental procedures for measurement of PAH accumulation in the slices were essentially the same as described elsewhere (18). Renal cortical slices weighing approximately $150 \mathrm{mg}$ were incubated at $37^{\circ} \mathrm{C}$ in $10 \mathrm{ml}$ of incubation medium containing $134 \mathrm{mM} \mathrm{NaCl}, 5.9 \mathrm{mM} \mathrm{KCl}, 1.5 \mathrm{mM}$ $\mathrm{CaCl}_{2}, 1.2 \mathrm{mM} \mathrm{MgCl}, 11.5 \mathrm{mM}$ glucose, $5.8 \mathrm{mM} \quad N$-2-hydroxyethyl-piperazine- $N^{\prime \prime}-2$ ethanesulfonic acid buffer (Hepes) $(\mathrm{pH} 7.4)$. $0.074 \mathrm{mM}$ PAH and $1 \%$ inulin, which was added to estimate the extracellular space of the slices. After incubation, the samples of the slices and medium were used for spectrophotometric analyses of $\mathrm{PAH}$ and inulin by the methods of Bratton and Marshall (19) and Roe et al. (20), respectively. PAH accumulated was expressed as the slice-tomedium concentration ratio (S/M) for $\mathrm{PAH}$.

Chemicals and statistics: Cisplatin was purchased from Sigma Chemical Co. (St. Louis, MO). DPPD was obtained from the Tokyo Kasei Kogyo Co., Ltd. (Tokyo, Japan) and dissolved in $99.6 \%$ methyl alcohol, which was added to the medium at the final concentration of $0.4 \%$. Other chemicals were of the highest purity available from commercial sources.

Data are expressed as means \pm S.E. Statistical analysis was done by analysis of variance. Significant differences at the level of $P<0.05$ were evaluated among multiple comparisons with Dunnett's test (21).

\section{Results}

Cisplatin-induced lipid peroxidation in slices: In vitro studies are useful for investigating whether cisplatin directly affects the production of lipid peroxidation in tissues. We used renal slices incubated with or without $2 \mathrm{mM}$ cisplatin at $37^{\circ} \mathrm{C}$ for various periods of time (Fig. 1). Although incubation of the slices with the drug gave no change in lipid peroxidation up to $30 \mathrm{~min}$, incubation

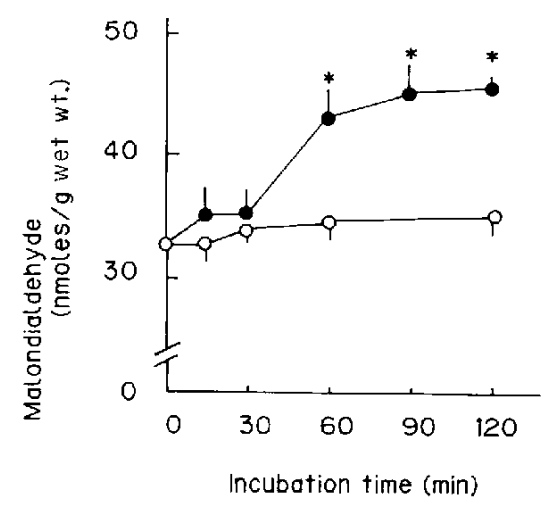

Fig. 1. Changes in malondialdehyde level in rat renal slices with (O) or without (O) $2 \mathrm{mM}$ cisplatin. Slices were incubated at $37^{\circ} \mathrm{C}$. Data represent the means \pm S.E. of four to seven experiments. ${ }^{*} P<0.01$ vs. the control value at time zero. 
Table 1. Effect of cisplatin and $N-N^{\prime}$-diphenyl-p-phenylenediamine (DPPD) on malondialdehyde level in rat renal slices

\begin{tabular}{ccc}
\hline \multicolumn{2}{c}{ Additions } & Malondialdehyde (nmoles/g tissue) \\
\hline None & & $33.13 \pm 1.04$ \\
Cisplatin & $(2 \mathrm{mM})$ & $47.42 \pm 0.68^{*}$ \\
Cisplatin & $(2 \mathrm{mM})$ & $32.46 \pm 0.63$ \\
+DPPD & $(1 \mu \mathrm{M})$ \\
DPPD alone & $(1 \mu \mathrm{M})$ & $28.13 \pm 1.60$ \\
\hline
\end{tabular}

Slices were incubated at $37^{\circ} \mathrm{C}$ for 120 min in medium containing the concentrations of cisplatin and DPPD shown. Data are the meanstS.E. of four experiments. " $P<0.01$ vs, the control with none.



Fig. 2. Effect of cisplatin concentration on malondialdehyde level in rat renal slices. Slices were incubated at $37^{\circ} \mathrm{C}$ for 120 min in medium containing the concentrations of cisplatin as shown in the figure. Data represent the means \pm S.E. of three to seven experiments. ${ }^{*} \mathrm{P}<0.05$ vs, the control without cisplatin. ${ }^{*} \mathrm{P}<0.01$ vs. the control without cisplatin.

for 60 min produced a significant increase. When incubation of the slices with cisplatin was carried out for $120 \mathrm{~min}$, the increase in lipid peroxidation was concentrationdependent (Fig. 2).

Incubation of the slices with neither compound resulted in the production of $33.13 \pm$ 1.04 nmoles of malondialdehyde/g tissue, which we took as the base-line value. The extra lipid peroxidation caused by $2 . \mathrm{mM}$ cisplatin was completely inhibited by $1 \mu \mathrm{M}$ DPPD (Table 1).

It has been reported that mannitol diuresis is useful in reducing the nephrotoxicity of cisplatin $(22,23)$. Figure 3 shows that the presence of $2 \mathrm{mM}$ mannitol in the incubation medium. which has been reported to be a concentration sufficient to scavenge free radicals generated by a nephrotoxic drug (24). almost completely inhibits the increase in

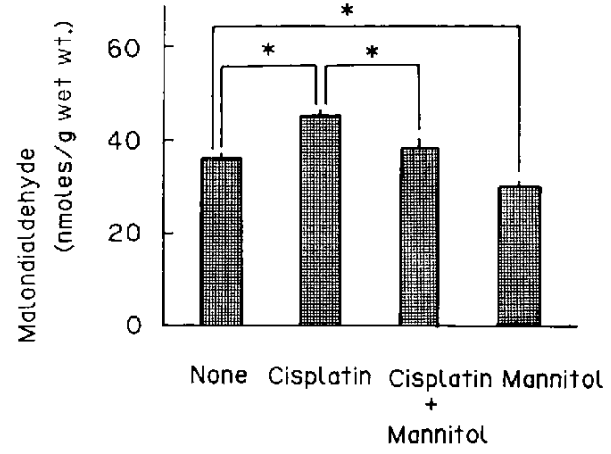

Fig. 3. Effect of cisplatin and mannitol on malondialdehyde level in rat renal slices. Slices were incubated at $37^{\circ} \mathrm{C}$ for $120 \mathrm{~min}$ in medium containing $2 \mathrm{mM}$ cisplatin and $2 \mathrm{mM}$ mannitol as indicated in the figure. Data represent the means $\pm S$.E. of four experiments. ${ }^{*}<<0.01$, when compared as shown.



Fig. 4. Effect of cisplatin and methionine on malondialdehyde level in rat renal slices. Slices were incubated at $37^{\circ} \mathrm{C}$ for $120 \mathrm{~min}$ in medium containing $2 \mathrm{mM}$ cisplatin and $4 \mathrm{mM}$ methionine as indicated in the figure. Data represent the means $\pm S$.E. of four experiments. $P<0.05,{ }^{*} P<0.01$.

lipid peroxidation caused by cisplatin. After incubation of the slices with cisplatin, the 
production of lipid peroxidation by cisplatin was further acceleraied in the presence of 4 $\mathrm{mM}$ methionine, although the addition of methionine (4 $\mathrm{mM}$ ) showed no effect on lipid peroxidation in the absence of cisplatin (Fig. 4). This acceleration by methionine was observed in concentrations of the amino acid from 0.5 to $10 \mathrm{mM}$ in a concentration dependent manner, but higher concentration of methionine itself $(10 \mathrm{mM})$ ) had a stimulatory effect on lipid peroxidation in the slices (data not shown).

Effects of cisplatin and DPPD on PAH accumulation in slices: We examined the effects of cisplatin with or without DPPD on PAH accumulation in rat renal cortical slices, which was measured for the evaluation of biochemical changes in transport ability of renal cells. Cisplatin caused a significant decrease in PAH accumulation in incubated slices (Fig. 5). This result was consistent with the results reported by other investigators $(25,26)$. The decrease in PAH accumulation in slices during $90 \mathrm{~min}$ of incubation with cisplatin was partially inhibited by DPPD. The antioxidant alone had no effect on PAH accumulation. Treatment of rats with DPPD $(0.5 \mathrm{~g} / \mathrm{kg}$, i.p. $)$ inhibited the decrease in PAH accumulation in the slices induced by



Fig. 5. Effect of cisplatin and $N$-N'diphenyl-pphenylenediamine (DPPD) on PAH accumulation in kidney cortical slices. Slices were incubated at $37^{\circ} \mathrm{C}$ for $90 \mathrm{~min}$ in medium containing cisplatin (1 $\mathrm{mM}$ ) and DPPD as indicated in the figure. Data represent the means $\pm S$.E. of seven experiments. ${ }^{*} \mathrm{P}<0.01 \mathrm{vs}$. the case "None". $\$ P<0.01$ vs. the case "Cisplatin".

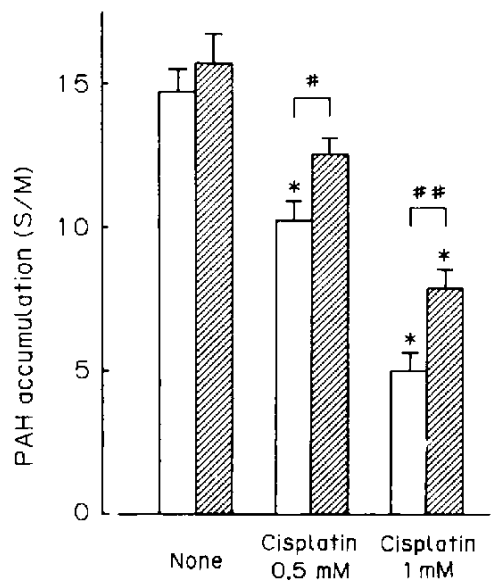

Fig. 6. Effect of $N$-N'diphenyl-p-phenylenediamine (DPPD) treatment on cisplatin-induced decrease of PAH accumulation in kidney cortical slices. Rats were treated with DPPD (WIIID. $0.5 \mathrm{~g} / \mathrm{kg}$, i.p.) or not ( $\square$ ) $24 \mathrm{hr}$ before removal of the kidneys. Control rats (not treated with DPPD) received corn oil i.p. PAH accumulation was measured after incubation of kidney cortical slices for $90 \mathrm{~min}$ at $37^{\circ} \mathrm{C}$. Data represent the means \pm S.E. of six experiments. ${ }^{*} \mathrm{P}<0.01$ vs, the value obtained without DPPD treatment labeled "None". \$P<0.05. $\ddagger \pm P<0,01$.

cisplatin (0.5 mM or $1 \mathrm{mM}$ ) (Fig. 6).

\section{Discussion}

Previous studies have demonstrated the effect of cisplatin injection into rats on malondialdehyde level in the kidney (8). indicating the increase in the malondialdehyde level at $72 \mathrm{hr}$ after cisplatin injection ( $5 \mathrm{mg} /$ $\mathrm{kg}$. i.p.). Pretreatment of rats with an antioxidant ( $\alpha$-tocopherol or DPPD) prevents the increase in the renal malondialdehyde level caused by cisplatin administration (8). The direct effect of cisplatin on renal tissues should be discussed in terms of the increase in malondialdehyde production. These in vitro experiments showed for the first time that cisplatin in the incubation medium increased the malondialdehyde level in the rat renal slices. The increase in the malondialdehyde level caused by cisplatin was concentration- and time-dependent, and it was prevented by the addition of DPPD. These results suggest that the increased lipid peroxidation with cisplatin may be due to a direct effect of the drug on the kidney and 
support the hypothesis that free radicals generated by cisplatin interact with membrane lipids to cause production of lipid peroxides. It is not yet clear, however, what free radical cisplatin generates.

Mannitol protects against the nephrotoxicity of cisplatin $(22,23)$ and scavenges hydroxyl radicals (27). The prevention by mannitol of the increase in malondialdehyde formation in renal slices caused by cisplatin in this study may arise from scavenging of reactive oxygen species by mannitol. Cisplatin nephrotoxicity monitored by blood urea nitrogen is increased by administration of methionine at the same time (28). The presence of methionine made the slices more susceptible to cisplatin-induced lipid peroxidation. The mechanism of such an effect of methionine on cisplatin-induced lipid peroxidation remains to be established. Mannitol and methionine may modify the nephrotoxicity of cisplatin by affecting the production of lipid peroxides.

The decrease in PAH accumulation caused by cisplatin was inhibited not only by the addition of DPPD in the medium but also by pretreatment with DPPD. Csallany and Draper showed that DPPD, following its administration to rats, is accumulated in the liver and skeletal muscle (29). However. they did not examine the distribution of DPPD in kidney. Ramsammy et al. (30) observed that injection of DPPD to rats reduces the increase in renal lipid peroxidation induced by aminoglycoside, suggesting that DPPD injected into rats may be distributed in the kidney. Preliminary experiments in our laboratory have shown that addition of DPPD to the medium does not prevent the in vitro inhibition of sodium-potassium-activated ATPase activity by cisplatin in rat renal microsomes. If there was a chemical interaction of cisplatin with DPPD, this would not be the case. Therefore, this inhibitory effect of DPPD on the decrease in PAH accumulation caused by cisplatin probably was not due to the chemical interaction of cisplatin with DPPD.

Platinum accumulates in the kidney and is found in many subcellular sites of the kidney, mostly in the cytosolic compartment, when cisplatin is injected to rats $(31,32)$. Rat renal cortical slices concentrate cisplatin in energy- and temperature-dependent systems from the incubation medium (33). The intracellular site at which cisplatin exerts its nephrotoxic effect is unknown. If an excess of free radicals, which overwhelm the cellular protective mechanisms, are formed close to plasma membranes by cisplatin, they may cause changes of membrane components such as lipids and protein and consequently change transport activity. The prevention by DPPD of the decrease in PAH accumulation caused by cisplatin may reflect the action of the drug on the membranes by involvement in the formation of free radicals.

Details of the mechanisms involved in the increase in lipid peroxidation in renal tissues caused by cisplatin are still unknown. Together with in vivo results that antioxidants which attenuate the increase in blood urea nitrogen caused by cisplatin (7) protect against the increase in lipid peroxidation caused by the drug (8), the findings in this study lead us to speculate that the nephrotoxic effect of cisplatin is associated at least in part with lipid peroxidation mediated by free radicals.

Acknowledgements: The authors thank Ms. Caroline Latta for help in preparing the manuscript and Ms. Eiko Ito, a student of our university, for technical assistance.

\section{References}

1 Rozencweig, M., Von Hoff, D.D., Slavik, M. and Muggia, F.M.: Cis-Diamminedichloroplatinum (II). A new anticancer drug. Ann. Intern. Med. 86, 803-812 (1977)

2 Blachley, J.D. and Hill, J.B.: Renal and electrolyte disturbances associated with cisplatin. Ann. Intern. Med. 95, 628-632 (1981)

3 Goldstein, R.S. and Mayor, G.H.: The nephrotoxicity of cisplatin. Life Sci. 32, 685-690 (1983)

4 Weiner, M.W. and Jacobs, C.: Mechanism of cisplatin nephrotoxicity. Fed. Proc. 42, 29742978 (1983)

5 Litterst, C.L.: Cisplatinum: A review, with special reference to celiular and molecular interactions. Agents Actions 15, 520-524 (1984)

6 McGinness, J.E., Proctor, P.H., Demopoulos, H.B., Hokanson, J.A. and Kirkpatrick, D.S.: Ameliaration of cis-platinum nephrotoxicity by orgotein (superoxide dismutase). Physiol. Chem. Phys. 10, 267-277 (1978) 
7 Sugihara, K. and Gemba, M.: Modification of cisplatin toxicity by antioxidants. Japan. J. Pharmacol. 40, 353-355 (1986)

8 Sugihara, K., Nakano, S., Koda, M., Tanaka, K., Fukuishi, N. and Gemba, M.: Stimulatory effect of cisplatin on production of lipid peroxidation in renal tissues. Japan. J. Pharmacol. 43, 247252 (1987)

9 Fridovich, I.: The biology of oxygen radicals. Science 201, 875-880 (1978)

10 Slater T.F.: Free-radical mechanisms in tissue injury. Biochem. J. 222, 1-15 (1984)

11 Kuo, C.-H., Maita, K., Sleight, S.D. and Hook, J.B.: Lipid peroxidation: A possible mechanism of cephaloridine-induced nephrotoxicity. Toxicol. Appl. Pharmacol. 67, 78-88 (1983)

12 Gastraunthaler, G., Pfaller, W. and Kotanko, P.: Glutathione depletion and in vitro lipid peroxidation in mercury or maleate induced acute renal failure. Biochem. Pharmacol. 32, 29692972 (1983)

13 Ramsammy, L., Ling. K.-Y,, Josepovitz, C., Levine, R. and Kaloyanides, G.J.: Effect of gentamicin on lipid peroxidation in rat renal cortex. Biochem. Pharmacol. 34, 3895-3900 (1985)

14 Paller, M.S., Hoidal, J.R. and Ferris, T.F.: Oxygen free radicals in ischemic acute renal failure in the rat. J. Clin. Invest. 74, 1156-1164 (1984)

15 Masuda, $Y$. and Murano, T.: Carbon tetrachlorideinduced lipid peroxidation of rat liver microsomes in vitro. Biochem. Pharmacol. 26, 22752282 (1977)

16 Cojocel, C., Laeschke, K.H., Inselmann, G. and Baumann, K.: Inhibition of cephaloridine-induced lipid peroxidation. Toxicology 35, 295-305 (1985)

17 Buege, J.A. and Aust, S.D.: Microsomal lipid peroxidation. Methods Enzymol. 52, 302-310 (1978)

18 Gemba, M., Tachibana, A., Sugihara, K., Hori, M. and Nakajima, M.: Inhibitory effect of lithium on $p$-aminohippurate transport in rat kidney cortex in vitro. Renal Physiol. 8, 179-188 (1985)

19 Bratton, A.C. and Marshall, E.K., Jr.: A new coupling component for sulfanilamide determination. J. Biol. Chem. 128, 537-550 (1939)

20 Roe, J.H., Epstein, J.H. and Goldstein, N.P.: A photometric method for the determination of inulin in plasma and urine. J. Biol. Chem. 178, 839-845 (1949)

21 Dunnett, C.W.: A multiple comparison procedure for comparing several treatments with a control. J. Am. Statist. Assoc. 50, 1096-1121 (1955)

22 Hayes, D., Cvitkovic, E., Golbey, R., Scheiner, E. and Krakoff, I.H.: Amelioration of renal toxicity of high dose cis-platinum diammine dichloride (CPDD) by mannitol induced diuresis. Proc. Am. Assoc. Cancer Res. and ASCO 17, 169 (1976)

23 Pera, M.F., Jr., Zook, B.C. and Harder, H.C.: Effects of mannitol or furosemide diuresis on the nephrotoxicity and physiological disposition of cis-dichlorodiammineplatinum-(Ii) in rats. Cancer Res. 39, 1269-1278 (1979)

24 Cojocel, C., Hannemann, J. and Baumann, K.: Cephaloridine-induced lipid peroxidation initjated by reactive oxygen species as a possible mechanism of cephaloridine nephrotoxicity. Biochim. Biophys. Acta 834, 402-410 (1985)

25 Guarino, A.M., Miller, D.S., Arnold, S.T., Pritchard, J.B., Davis, R.D., Urbanek, M.A., Miller, T.J. and Litterst, C.L.: Platinate toxicity: Past, present. and prospects: Cancer Treat. Rep. 63, 14751483 (1979)

26 Goldstein, R.S., Noordewier, B., Bond, J.T., Hook, J.B. and Mayor, G.H.: cis-Dichlorodiammineplatinum nephrotoxicity: Time course and dose response of renal functional impairment. Toxicol. Appl. Pharmacol. 60, 163-175 (1981)

27 Gutteridge, J.M.C: Lipid peroxidation initiated by superoxide-dependent hydroxyl radicals using complexed iron and hydrogen peroxide. FEBS Lett. 172, 245-249 (1984)

28 Wesley Alden, W. and Repta, A.J.: Exacerbation of cisplatin-induced nephrotoxicity by methionine. Chem. Biol. Interact. 48, 121-124 (1984)

29 Saari Csallany, A. and Draper, H.H.: Determination of $N, N^{\prime}$-diphenyl-p-phenylenediamine in animal tissues. Proc. Soc. Exp. Biol. Med. 104, 739-742 (1960)

30 Ramsammy, L.S., Josepovitz, $C_{1,}$ Ling, K.-Y., Lane, B.P. and Kaloyanides, G.J.: Effects of diphenyl-phenylenediamine on gentamicininduced lipid peroxidation and toxicity in rat renal cortex. J. Pharmacol. Exp. Ther. 238, 83$88(1986)$

31 Choie, D.D., Del Campo, A.A. and Guarino, A.M.: Subcellular localization of cis-dichlorodiammineplatinum (li) in rat kidney and liver. Toxicol. Appl. Pharmacol. 55, 245-252 (1980)

32 Sharma, R.P. and Edwards, I.R.: cis-Platinum: Subcellular distribution and binding to cytosolic ligands. Biochem. Pharmacol. 32, 2665-2669 (1983)

33 Safirstein, R., Miller, P. and Guttenplan, J.B.: Uptake and metabolism of cisplatin by rat kidney. Kidney Int. 25, 753-758 (1984) 